\title{
Bronchoscopic management of asthma, COPD and emphysema
}

\author{
Jeanne Marie Perotin ${ }^{1,2}$, Maxime Dewolf ${ }^{1}$, Claire Launois ${ }^{1}$, Valérian Dormoy (i) $^{2}$ \\ and Gaëtan Deslee ${ }^{1,2}$
}

Number 6 in the Series "Interventional pulmonology"

Edited by David Feller-Kopman and Hervé Dutau

Affiliations: ${ }^{1}$ Dept of Pulmonary Medicine, University Hospital of Reims, Reims, France. ${ }^{2}$ INSERM P3Cell UMR-S1250, SFR CAP-SANTE, University of Reims Champagne Ardenne, Reims, France.

Correspondence: Gaëtan Deslee, Dept of Pulmonary Medicine, University Hospital of Reims, 45 rue CognacqJay, 51092 Reims Cedex, France. E-mail gdesleelachu-reims.fr

@ERSpublications

Many therapeutic bronchoscopic techniques have been recently developed including bronchial thermoplasty in asthma, and valves, coils and thermal vapor ablation in emphysema, and targeted lung denervation in COPD https://bit.ly/2VhMsDI

Cite this article as: Perotin JM, Dewolf M, Launois C, et al. Bronchoscopic management of asthma, COPD and emphysema. Eur Respir Rev 2021; 30: 200029 [https://doi.org/10.1183/16000617.0029-2020].

ABSTRACT In recent years, many bronchoscopic techniques have been developed in chronic obstructive airway inflammatory diseases, including asthma, COPD and emphysema. The main techniques with available data from randomised controlled trials are: 1) bronchial thermoplasty in asthma; 2) valves, coils and thermal vapor ablation in emphysema; and 3) targeted lung denervation in COPD. The objectives of this article are to describe the levels of evidence for efficacy and safety, long-term follow-up results beyond 1 year, and current recommendations for clinical practice from international guidelines for each technique.

\section{Introduction}

The field of interventional bronchoscopy in chronic obstructive airway inflammatory diseases including asthma, COPD and emphysema has greatly evolved in recent years. Many bronchoscopic techniques have been developed and assessed initially in nonrandomised studies, with promising results raising hope and excitement for the management of these challenging patients. In the past 15 years, several randomised controlled trials (RCT) have been conducted allowing the ability to determine the levels of evidence for each technique. In this article, we focus on bronchoscopic techniques with available data from RCTs including: 1) bronchial thermoplasty in asthma; 2) valves, coils and thermal vapor ablation in emphysema; and 3) targeted lung denervation in COPD.

The aims of this article are to describe the following for each technique: 1) the levels of evidence for efficacy and safety based on RCTs results; 2) long-term follow-up results beyond 1 year from RCTs or

Provenance: Commissioned article, peer reviewed.

Previous articles in this series: No. 1: DeMaio A, Sterman D. Bronchoscopic intratumoral therapies for nonsmall cell lung cancer. Eur Respir Rev 2020; 29: 200028. No. 2: Chandrika S, Yarmus L. Recent developments in advanced diagnostic bronchoscopy. Eur Respir Rev 2020; 29: 190184. No. 3: Kniese CM, Musani AI. Bronchoscopic treatment of inoperable nonsmall cell lung cancer. Eur Respir Rev 2020; 29: 200035. No. 4: Rosell A, Stratakos G. Therapeutic bronchoscopy for central airway disease. Eur Respir Rev 2020; 29: 1900178. No. 5: Bedawi EO, Guinde J, Rahman NM, et al. Advances in pleural infection and malignancy. Eur Respir Rev 2021; 30: 200002.

Received: 31 Jan 2020 | Accepted after revision: 15 June 2020

Copyright CERS 2021. This article is open access and distributed under the terms of the Creative Commons Attribution Non-Commercial Licence 4.0. 
"real-life" studies when available; and 3) current recommendations for clinical practice from international guidelines.

\section{Asthma}

Bronchial thermoplasty is a bronchoscopic procedure performed under general anaesthesia or conscious sedation that delivers controlled thermal energy to the airway wall during a series of bronchoscopy procedures (three procedures in the main RCTs), resulting in a prolonged reduction in airway smooth muscle mass $[1,2]$ thought to improve bronchoconstriction in asthma [3].

\section{Levels of evidence}

The results of three RCTs have been published [4-6] and long-term analysis 5 years after treatment are available for two of these RCTs (table 1) [7, 8].

The AIR study [4] included 112 subjects randomised to bronchial thermoplasty $(\mathrm{n}=56)$ or medical management (control, $\mathrm{n}=56$ ). Subjects had moderate to severe asthma, with forced expiratory volume in $1 \mathrm{~s}$ $\left(\mathrm{FEV}_{1}\right) 60-85 \%$ of the predicted value and worsening of symptoms on long-acting $\beta$-agonist (LABA) withdrawal. No sham procedure was performed. Outcome assessment at 12 months identified a decrease in the number of mild exacerbations, improvement in peak expiratory flow (PEF), asthma control questionnaire (Asthma Quality of Life Questionnaire (AQLQ) and Asthma Control Questionnaire (ACQ)) scores, symptom-free days and rescue medication use. No significant changes in $\mathrm{FEV}_{1}$, airway responsiveness (AHR) or number of severe exacerbations were observed.

The RISA trial [5] focused on people with severe asthma, defined as requiring high-dose inhaled corticosteroids (ICS) and LABAs with or without oral prednisone, leukotriene modifiers or theophylline. Other inclusion criteria were $\mathrm{FEV}_{1} \geqslant 50 \%$ pred and AHR. Subjects were randomised to bronchial thermoplasty $(\mathrm{n}=15)$ or medical management (control, $\mathrm{n}=17)$. In the bronchial thermoplasty group, after a 16-week ICS dose-stability phase, ICS dose was reduced. Assessment 52 weeks after treatment identified a reduction in rescue medication use and improvement in ACQ and AQLQ scores. FEV F $_{1}$ aHR were not modified.

The AIR2 trial [6] included 288 people with severe asthma (high-dose ICS+LABA) randomised to bronchial thermoplasty $(n=190)$ or sham control $(n=98)$. Subjects had $\mathrm{FEV}_{1} \geqslant 60 \%$, AHR and AQLQ $\leqslant 6.25$. Outcome assessment performed 12 months after treatment showed improved AQLQ score and decrease in the number of severe exacerbations, emergency department visits and days lost from work/ school. No change in PEF, ACQ score, number of symptom-free days, frequency of rescue medication use and rates of hospitalisation was noted. Of note, a substantial sham effect was described in this study including a clinically meaningful improvement in AQLQ score $(\geqslant 0.5)$ in $64 \%$ of the sham group $(79 \%$ in bronchial thermoplasty group, posterior probability of superiority 99.6\%) and a mean improvement of 1.16 in AQLQ in the sham group (1.35 in the bronchial thermoplasty group, posterior probability of superiority 96.0\%). This unexpected placebo effect was discussed as resulting from preconceived expectations and the care and attention provided by the study staff.

In all three RCTs, bronchial thermoplasty procedure was associated with frequent immediate and transient worsening of asthma symptoms [4-6]. Consolidation, atelectasis and upper or lower respiratory tract infections were also reported.

Overall, these three RCTs suggest a beneficial impact of bronchial thermoplasty on asthma control and quality of life with an acceptable safety profile. However, one limitation is that a sham group has been conducted in only one study, which showed a substantial sham effect. The criteria of inclusion in these three studies are somewhat heterogeneous, but selected mainly severe asthma with relatively similar patients' characteristics. The primary end-points are very heterogeneous based on exacerbation, safety or asthma control questionnaires, making the comparison between these RCTs difficult.

The grading of recommendations, assessment, development and evaluations (GRADE) for bronchial thermoplasty from these three RCTs is moderate with a low risk of bias, low risk of imprecision, consistency of efficacy results but limited to three RCTs, no evident indirectness, and no known publication bias.

\section{Long-term follow-up results}

Long-term analysis (5 years) of the AIR study [7] included 45 subjects in the bronchial thermoplasty group and 24 controls and identified improvement of AHR up to 3 years after bronchial thermoplasty. No clinical complications, increase in hospitalisations or emergency department visits, or increased adverse events beyond 1 year were noted, whereas $\mathrm{FEV}_{1}$ remained stable. The analysis of the AIR2 study performed 5 years after treatment included 162 subjects in the bronchial thermoplasty group [8] and identified a sustained reduction in the number of exacerbations and emergency department visits. The authors noted an $18 \%$ 
TABLE 1 Characteristics of randomised controlled trials

\begin{tabular}{llcl} 
Study [Ref.] & Subjects N & Main inclusion criteria & $\begin{array}{c}\text { Control } \\
\text { group }\end{array}$ \\
\hline
\end{tabular}

\section{Asthma: BT} AIR [4]

RISA [5]

AIR2 [6]

\section{Emphysema: endobronchial lung volume reduction Valve: EBV or SVS} BeLieVeR-HIFI [9]

STELVIO [10]

IMPACT [11]

LIBERATE [13]

REACH [14]

EMPROVE [15]

Coils RESET [16]
BT: 56; Moderate/severe asthma, worsening control on Usual Frequency of mild exacerbations at

control: $56 \quad$ LABA withdrawal, $\mathrm{FEV}_{1} 60-85 \% \quad$ care 3, 6, 12 months

Primary end-point results: change in frequency per subject per week from baseline: $-0.16 \pm 0.37$ in BT group versus $0.04 \pm 0.29$ in control group, $p=0.005$
BT: 15;
Severe asthma, $\mathrm{FEV}_{1} \geqslant 50 \%$
Usual
Safety: adverse events

control: 17

care

Primary end-point results: 136 respiratory adverse events in BT group ( $49 \%$ mild, $41 \%$ moderate, $10 \%$ severe) versus 57 respiratory adverse events in control group (49\% mild, 47\% moderate, $4 \%$ severe)
BT: 196;
Severe asthma, $\mathrm{FEV}_{1} \geqslant 60 \%$
Sham
Integrated $A Q L Q$ score at 6, 9, control: 101 12 months

Primary end-point results: change in integrated AQLQ score: $1.35 \pm 1.10$ in BT group versus $1.16 \pm 1.23$ in control group; posterior probability of superiority $96.0 \%$
EBV: 25;

control: 25

Primary en $-p$ $(p=0.03)$

EBV: 34 ; control: 34
$\mathrm{FEV}_{1}<50 \%, \mathrm{RV}>150 \%$, heterogeneous, collateral Sham ventilation assessed by $\mathrm{CT}$ scan (visual)
Percentage change FEV 1 at 3 months $\begin{aligned} & \text { by CT scan (visual) and Chartis } \\ & \text { Primary end-point results: } \mathrm{FEV}_{1}: 20.9 \%(95 \% \mathrm{Cl} 11.1-30.7) \text { in EBV group versus } 3.1 \%(95 \% \mathrm{Cl}-0.4 \text { to } 6.6) \text { in }\end{aligned}$ control group, difference between group 17.8\% (95\% Cl 7.6-28.0), p=0.001; FVC: $18.3 \%$ (95\% Cl 9.3-27.3) in EBV group versus $4.0 \%(95 \% \mathrm{Cl}-0.7-8.6)$ in control group, difference between group $14.4 \%(95 \% \mathrm{Cl} 4.4-24.3)$, $\mathrm{p}=0.005$; $6 \mathrm{MWT}$ : $19.6 \%(95 \% \mathrm{Cl}$ 10.4-28.9) in EBV group versus $-3.6(95 \% \mathrm{Cl}-6.9-0.4)$, difference between group 23.3 (95\% Cl 13.6-32.9), $\mathrm{p}<0.001$

EBV: $43 ; \quad 15 \%<\mathrm{FEV}_{1}<45 \%$; RV $>200 \%$, homogeneous, Usual Percentage change $\mathrm{FEV}_{1}$ at control : $50 \quad$ collateral ventilation assessed by Chartis care 3 months

Primary end-point results: $\mathrm{FEV}_{1}: 13.7 \pm 28.2 \%$ in the EBV group versus $-3.2 \pm 13.0 \%$ in control group; mean difference between groups of $17.0 \%(95 \% \mathrm{Cl} 8.1-25.8 \%, \mathrm{p}=0.0002)$

EBV: $65 ; \quad 15 \%<\mathrm{FEV}_{1}<45 \%$; RV $>180 \%$, heterogeneous, Usual Percentage subjects with $\geqslant 12 \%$ control: 32 collateral ventilation assessed by Chartis care improvement from baseline in

Primary end-point results: $55.4 \%$ in the EBV group; $6.5 \%$ in the control group ( $p=0.001)$ $\mathrm{FEV}_{1}$ at 3 months

EBV: 128 ; $\quad 15 \%<\mathrm{FEV}_{1}<45 \%$; RV $>175 \%$, heterogeneous, Usual Percentage subjects with $\geqslant 15 \%$ control: 62 collateral ventilation assessed by Chartis care improvement from baseline in $\mathrm{FEV}_{1}$ at 12 months

Primary end-point results: $47.7 \%$ in the EBV group; $16.8 \%$ in the control group; between-group absolute difference of $31.0(95 \% \mathrm{Cl} 18.0-43.9 \% ; \mathrm{p}=0.001)$

SVS: 66; $\quad \mathrm{FEV}_{1} \leqslant 45 \%, \mathrm{RV} \geqslant 150 \%$, heterogeneous, intact Usual Difference between groups in control: 33 interlobar fissure assessed by CT scan (visual) care mean change $\mathrm{FEV}_{1}$ at 3 months Primary end-point results: $F E V_{1} 0.104 \pm 0.18 \mathrm{~L}$ in SVS group versus $0.003 \pm 0.15 \mathrm{~L}$ in control group, $p=0.001$

SVS: $113 ; \quad \mathrm{FEV}_{1}<45 \%, \mathrm{RV}>150 \%$, heterogeneous, intact Usual Difference between groups in control: 59 interlobar fissure assessed by CT scan (visual) care mean change FEV1 at 6 months Primary end-point results: $\mathrm{FEV}_{1} 0.099 \mathrm{~L}(95 \% \mathrm{Cl} 0.069-0.128)$ in SVS group; $0.002 \mathrm{~L}(95 \% \mathrm{Cl} 0.030-0.026)$; between-group difference $0.101 \mathrm{~L}(95 \% \mathrm{Cl} 0.060-0.141)$

Coil: 23; $\quad \mathrm{FEV}_{1} \leqslant 45 \%$, homogeneous and heterogeneous Usual

Difference in quality of life (SGRQ) control: 24

care between both groups at 90 days

Primary end-point results: SGRQ: $-8.11(-13.83--2.39)$ in coil group; $0.25(-5.58-6.07)$ in control group; between-group difference: $-8.36(-16.24--0.47), p=0.04$ 
TABLE 1 Continued

Study [Ref.] Subjects N $\quad$ Main inclusion criteria $\quad \begin{gathered}\text { Control } \\ \text { group }\end{gathered}$ Primary end-point

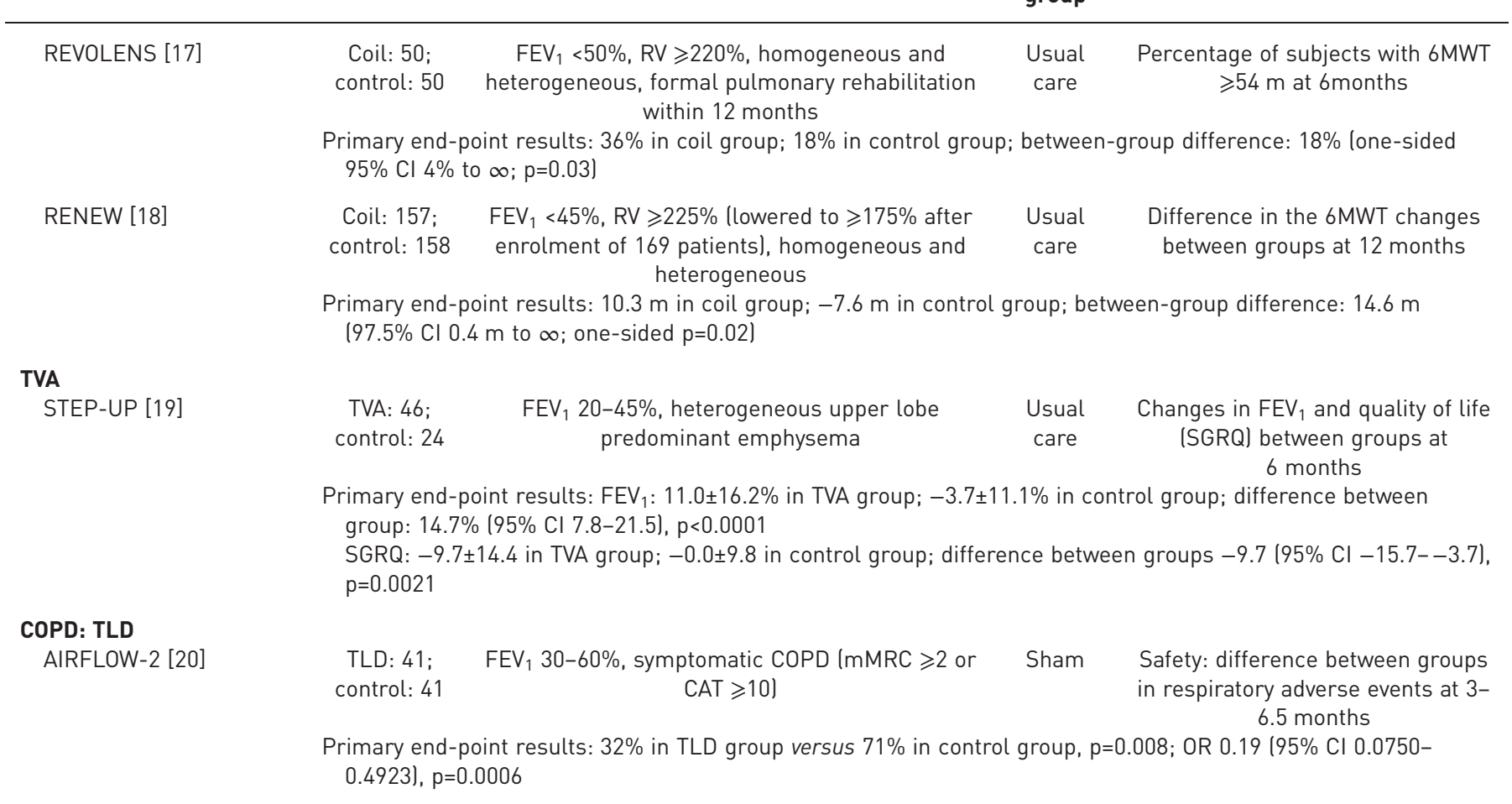

BT: bronchial thermoplasty; EBV: endobronchial valve; SVS: Spiration valve system; TVA: thermal vapor ablation; TLD: targeted lung denervation; LABA: long-acting $\beta$-agonist; $\mathrm{FEV}_{1}$ : forced expiratory volume in $1 \mathrm{~s} ; \mathrm{AQLQ}$ : Asthma Quality of Life Questionnaire; RV: residual volume; CT: computed tomography; FVC: forced vital capacity; 6MWT: 6-min walk test; SGRQ: St George's Respiratory Questionnaire; mMRC: modified Medical Research Council dyspnoea score; CAT: COPD Assessment Test.

reduction in ICS daily dose with stable pre-bronchodilator $\mathrm{FEV}_{1}$ values. Computed tomography (CT) scan performed at year 5 ( $\mathrm{n}=93$ subjects) showed no abnormalities attributable to bronchial thermoplasty.

A post-US Food and Drug Administration (FDA) approval study conducted in the USA (the PAS2 study) included 190 patients showing a decrease in the number of severe exacerbations, emergency department visits and hospitalisation at 3 years [21].

Long-term analysis of bronchial thermoplasty impact beyond 1 year relies on data from two RCTs and one real-life study. These results suggest long-term safety and persistence of benefits.

\section{Current recommendations}

International recommendations do not recommend bronchial thermoplasty as routine management of severe asthma. The European Respiratory Society/American Thoracic Society guidelines of severe asthma management [22] do recommend that bronchial thermoplasty is performed in adults with severe asthma only in the context of an Institutional Review Board-approved independent systematic registry or a clinical study. The 2020 Global Initiative for Asthma guidelines [23] specify that bronchial thermoplasty is a potential treatment option at Step 5 in some countries for adult patients whose asthma remains uncontrolled despite optimisation of asthma therapy and referral to a severe asthma specialty centre (Evidence B), and should be performed in adults with severe asthma only in the context of an independent Institutional Review Board-approved systematic registry or a clinical study. Both international guidelines base these recommendations on the limited evidence for bronchial thermoplasty efficacy and long-term safety in severe asthma.

\section{Emphysema}

The aim of bronchoscopic techniques in emphysema is to reduce hyperinflation. The three main techniques with available robust RCT results are endobronchial valves, coils and thermal vapor ablation. 


\section{Endobronchial valves}

Endobronchial one-way valves are placed through fibreoptic bronchoscopy in segmental bronchi in a targeted lobe. Valves allow expiratory flow of air while blocking inspiratory flow in the targeted lobe, therefore resulting in the reduction of lobar volume which can eventually lead to full lobar collapse. Among several endobronchial valves developed so far, two valves only have been assessed in RCTs: the Zephyr one-way endobronchial valve (EBV) (PulmonX, Redwood City, CA, USA) and the Spiration valve system (SVS) (Olympus, Redmond, WA, USA) previously known as an intra-bronchial valve. Early nonrandomised studies and post hoc analyses from RCTs clearly indicated that both collateral ventilation and nonoptimal placement of the valves with incomplete treatment of a targeted lobe were associated with poor efficacy results. These results lead to selecting patients based on the absence of collateral ventilation assessed by CT scan and/or endobronchial measurements using the Chartis system (PulmonX). In this review, we will focus on RCTs with inclusion criteria including the absence of collateral ventilation and with complete lobar treatment by valves.

\section{Levels of evidence}

Seven RCTs selecting patients with no or little collateral ventilation and complete lobar treatment have been conducted (table 1). Five RCTs used the EBV system, and two used the SVS system. All these RCTs used unilateral occlusion of a targeted lobe by valves.

\section{EBV system}

The BeLieVeR-HIFI study is a single-centre sham-controlled study conducted in the UK that included 50 patients (EBV: $\mathrm{n}=25$; sham: $\mathrm{n}=25$ ) [9]. The main inclusion criteria were $\mathrm{FEV}_{1}<50 \%$, residual volume (RV) $>150 \%$, 6-min walk test $(6 \mathrm{MWT})<450 \mathrm{~m}$, modified Medical Research Council (mMRC) dyspnoea score $\geqslant 3$, heterogeneous emphysema and intact interlobar fissure based on CT scan assessment. The primary end-point of $\mathrm{FEV}_{1}$ change at 3months, was significantly higher in the EBV group than in the sham group (table 1). Among the four patients in the EBV group with collateral ventilation detected using the Chartis system despite intact fissure diagnosed by CT scan, none had an improvement in $\mathrm{FEV}_{1}$, highlighting the importance of absence of collateral ventilation for EBV effectiveness. Secondary end-points demonstrated a significant improvement in the 6MWT, but no significant changes for the mMRC scale, St George's Respiratory Questionnaire (SGRQ) score, or RV at 3 months. Follow-up data, including patients treated by EBV from the sham group $(n=12)$ and the initial EBV group with no collateral ventilation based on Chartis assessment reported much better results with an improvement from baseline in $\mathrm{FEV}_{1}, \mathrm{RV}, 6 \mathrm{MWT}$ and SGRQ score [24].

The STELVIO study is a single-centre randomised study conducted in the Netherlands, including 68 patients (EBV: $\mathrm{n}=34$; usual care: $\mathrm{n}=34$ ) [10]. The main inclusion criteria were $\mathrm{FEV}_{1}<60 \%, \mathrm{RV}>150 \%$, heterogeneous emphysema, and absence of collateral ventilation assessed by Chartis. Significant improvement was found at 6 months for $\mathrm{FEV}_{1}$, forced vital capacity (FVC) and 6MWT in the EBV group compared to the usual care group. Results at 1 year for the EBV group and the control group who crossed over were reported for 40 out of 64 patients treated by EBV [25]. Significant improvements from baseline were reported for $\mathrm{FEV}_{1}, \mathrm{RV}, 6 \mathrm{MWT}$ and the SGRQ score. Two deaths occurred during the 1-year follow-up period.

The IMPACT study is a multicentre study conducted in Germany, the Netherlands and Austria, including 93 patients (EBV: $n=43$; usual care: $n=50$ ) with inclusion criteria including $15 \%<\mathrm{FEV}_{1}<45 \%$, $\mathrm{RV}>200 \%$, homogeneous emphysema, and absence of collateral ventilation assessed by Chartis [11]. Significant improvement was found for $\mathrm{FEV}_{1}$ change at 3months relative to baseline in the EBV group $(+13.7 \%$ versus $-3.2 \%)$. Secondary end-points at 3 months also demonstrated improvement in the EBV group compared to the control group for RV, SGRQ score and 6MWT.

The TRANSFORM study is a multicentre study conducted in the UK, Netherlands, Belgium, Sweden, France and Germany, including 97 patients (EBV: $n=65$; control: $n=32$ ). The main inclusion criteria were $15 \%<\mathrm{FEV}_{1}<45 \%, \mathrm{RV}>180 \%$, heterogeneous emphysema and absence of collateral ventilation assessed by Chartis [12]. The primary end-point was the percentage of subjects with an improvement of $\operatorname{FEV}_{1} \geqslant 12 \%$ in the EBV group compared to control at 3 months, which was achieved by $55.4 \%$ in the EBV group and $6.5 \%$ in the control group. At 6 months, improvements were maintained in the EBV group for $\mathrm{FEV}_{1}$. Between-group differences at 6 months were significant for RV, 6MWT and SGRQ.

The LIBERATE study is a multicentre study conducted in the USA, Brazil, UK, and the Netherlands, including 190 patients (EBV: $\mathrm{n}=128$; usual care: $\mathrm{n}=62$ ). The main inclusion criteria were $15 \%<\mathrm{FEV}_{1}<45 \%$, RV $>175 \%$, heterogeneous emphysema and absence of collateral ventilation assessed by Chartis [15]. The primary end-point was the percentage of patients with a $\mathrm{FEV}_{1} \geqslant 15 \%$ from baseline at 12 months, which 
was achieved in $47.7 \%$ in the EBV group and $16.8 \%$ in the control group. Secondary end-points at 12 months showed a significant between-group difference for $\mathrm{FEV}_{1}, \mathrm{RV}, 6 \mathrm{MWT}$ and the SGRQ score.

All five RCTs using the EBV system reported pneumothorax as a major adverse event, occurring from $8 \%$ to $33.2 \%$ in the EBV groups [9-13] and most frequently in the first 30-45 days following treatment [12, 13]. The TRANSFORM study precisely described the management of pneumothorax: intervention and/or hospitalisation in 14 out of 19 subjects, observation in eight cases, placement of chest drain in 11 cases, and surgery in one case [12]. Pneumothorax-related deaths were reported in three cases in the LIBERATE study and one case in the TRANSFORM study $[12,13]$. Overall, valve removal or replacement was reported in $8-32 \%$ of cases [9-13].

\section{SVS system}

The REACH study is a multicentre study conducted in China including 99 patients (SVS: $n=66$; usual care: $n=33$ ) [14]. The main inclusion criteria were $\mathrm{FEV}_{1} \leqslant 45 \%, \mathrm{RV} \geqslant 150 \%$, heterogeneous emphysema, and intact interlobar fissure assessed visually by CT scan. Changes in $\mathrm{FEV}_{1}$ at 3 months were higher in the SVS group compared to the control group (table 1).

The EMPROVE study is a multicentre study conducted in the USA and Canada, including 172 patients (SVS: $\mathrm{n}=113$; usual care: $\mathrm{n}=59$ ) [15]. The main inclusion criteria were $\mathrm{FEV}_{1} \leqslant 45 \%$, $\mathrm{RV} \geqslant 150 \%$, heterogeneous emphysema, and intact interlobar fissure assessed visually by CT scan $(\geqslant 90 \%$ complete fissure with no segmental vessels crossing from adjacent lobes). The primary end-point analysis at 6 months showed a higher $\mathrm{FEV}_{1}$ in the SVS group when compared with the control group. This difference in $\mathrm{FEV}_{1}$ was maintained at 12 months. At 6 months, the SVS group exhibited significant improvements in RV and SGRQ score but not for the 6MWT.

The rate of pneumothorax at 6 months in the SVS groups was $7.6 \%$ in the REACH study, and $25.7 \%$ in the EMPROVE study, including $14.2 \%$ serious pneumothorax defined as requiring surgical intervention or prolonged air leak for $>7$ days $[14,15]$. As described with the EBV system, most of the pneumothorax occurred early after procedure: 66\% within 3 days after SVS procedure [15].

Overall, seven RCTs assessing valves (five for EBV, two for SVS) demonstrated clinically significant improvements at 3,6 or 12 months with an acceptable safety profile. Pneumothorax is a frequent adverse event requiring close monitoring of patients for at least 3 days post-procedure by experienced teams able to rapidly treat pneumothorax. In some patients, additional procedures for replacement or removal of valves are needed.

Regarding these seven RCTs, only one study included a sham group for control, with no effect on the primary end-point based on percent change in $\mathrm{FEV}_{1}$ at 3 months. The inclusion criteria were relatively homogeneous, selecting patients with high hyperinflation and none-to-little collateral ventilation. The primary end-points were somewhat heterogeneous regarding the time-points with assessments at 3 months for four RCTs, 6 months for two RCTs and 12months for one RCT. 3-month time-point results have to be considered cautiously in a chronic respiratory disease such as emphysema. FEV $_{1}$ was included in all RCTs as the primary end-point, alone in six RCTs and in combination with FVC and 6MWT in one study. FEV was $^{2}$ analysed as difference in percent or absolute value changes between groups, or using cut-off of $12 \%$ or $15 \%$.

The GRADE level of evidence for valves from these seven RCTs is high with a low risk of bias, a low risk of imprecision, consistency in efficacy results, no evident indirectness, and no known publication bias.

\section{Long-term follow-up results}

Despite many RCTs assessing valves in severe emphysema, data beyond 1 year are sparse.

A retrospective study assessed 10-year survival in 19 patients treated by EBV comparing patients with or without atelectasis assessed by CT scan at 1 month post-procedure [26]. In the atelectasis group, two $(40 \%)$ of five patients were still alive at 10 years compared with two (14\%) out of 14 patients in the group without atelectasis.

A study assessed 38 patients treated by EBV in clinical trials in Groningen (the Netherlands) who were invited for voluntary annual visit [27]. Data were available for 27 patients at 2 years and 22 patients at 3 years. At 2 years, significant improvement compared to baseline was found for RV, mMRC scale and SGRQ score. At 3 years, only the mMRC dyspnoea scale remained statistically improved. The rate of pneumothorax was $6 \%$ during the first year, which was lower than that reported in RCTs probably because of a selection bias excluding patients with early valve removal. Of note, no other pneumothorax occurred during the second and third year of follow-up. 
A retrospective real-life study assessed the outcome after EBV treatment in 256 patients treated at Heidelberg (Germany) [28]. 2- and 3-year follow-up visits were available in 100 and 66 patients, respectively. At 2 years, significant improvement was shown for RV $(-0.35 \mathrm{~L})$ and total lung capacity $(-0.38 \mathrm{~L})$, but not for $\mathrm{FEV}_{1}$ and $6 \mathrm{MWT}$. At 3 years, only mMRC scale change $(-0.5)$ was statistically improved. Patients with complete lobar atelectasis exhibited superior results at 3 years for $\mathrm{FEV}_{1}$, RV and 6MWT. Regarding safety, pneumothorax occurred in $22 \%$ of the patients, with $86 \%$ of these patients requiring chest tube insertion, and $41 \%$ required valve removal. During the 3 -year follow-up, all valves were permanently removed in $24.6 \%$ of the patients.

\section{Current recommendations}

The 2020 Global Initiative for Chronic Obstructive Lung Disease (GOLD) report indicates that valves can be considered when available in both heterogeneous and homogeneous emphysema, with no collateral ventilation (Evidence A) [29]. Recommendations from an international expert panel updated in 2019 stated that valves reached the evidence level to be used outside clinical trials in heterogeneous or homogeneous emphysema without collateral ventilation [30]. The US FDA approved valves in routine clinical practise in 2019. Valves are also approved in many countries in Europe and Asia.

\section{Coils}

Endobronchial shape-memory nitinol coils (BTG/PneumRx, Mountain View, CA, USA) are devices inserted into subsegmental bronchi to induce lung volume reduction and enhance lung recoil. Under general anaesthesia, approximately 10 coils (sizes 100,125 or $150 \mathrm{~mm}$ ) are placed in a targeted lobe through a catheter inserted in a bronchoscope with deployment guided by fluoroscopy. A contralateral treatment is usually performed 1 to 3 months after the first procedure.

\section{Levels of evidence}

Three RCTs assessed endobronchial coils in severe emphysema (table 1).

The RESET study is a multicentre study conducted in the UK which included 47 patients allocated to coil treatment $(n=23)$ or usual care $(n=24)$ [16]. The main inclusion criteria were a post-bronchodilator $\mathrm{FEV}_{1}$ $\leqslant 45 \%$, mMRC scale $\geqslant 2$, and either homogeneous or heterogeneous emphysema. A significant improvement was noted for SGRQ score, the primary end-point, at 90 days in the coil group compared to the control group. Secondary end-points analyses also showed significant between-group differences for $\mathrm{FEV}_{1}, \mathrm{RV}$ and the 6MWT. 12-month follow-up results from the RESET study, including both the cross-over and initial coil groups $(n=45)$, showed an improvement from baseline for the SGRQ score, $\mathrm{FEV}_{1}$ and the 6MWT.

The REVOLENS study is an independent multicentre study conducted in France that included 100 patients (coil group: $n=50$; usual care: $n=50$ ) [17]. The main inclusion criteria were a post-bronchodilator $\mathrm{FEV}_{1}<50 \%$ and $\mathrm{RV} \geqslant 220 \%$, and formal pulmonary rehabilitation within the previous 12 months. The population exhibited very severe airflow limitation and hyperinflation, and mainly homogeneous emphysema. The primary end-point, which was the improvement of at least $54 \mathrm{~m}$ in the $6 \mathrm{MWT}$ at 6 months compared to baseline was achieved. Mean between-group changes at 12 months were significant for $\mathrm{FEV}_{1}$ and SGRQ score.

The RENEW study is a multicentre study conducted in the USA and Europe, which included 315 patients randomised into the coil group $(n=157)$ or usual care group $(n=158)$ [18]. The main inclusion criteria were a post-bronchodilator $\mathrm{FEV}_{1}<45 \%$ and $\mathrm{RV} \geqslant 225 \%$ which was lowered to $\geqslant 175 \%$ after enrolment of 169 patients. Overall, the population exhibited severe hyperinflation and predominant homogeneous emphysema. The primary end-point which was the difference in the 6MWT between baseline and 12 months in both groups was achieved. Between-group differences at 12 months for effectiveness outcomes were significant for $\mathrm{FEV}_{1}$ and SGRQ. A post hoc analysis of the RENEW study identified that significant hyperinflation (RV $>200 \%$ ) and CT analyses including a quantitative assessment to identify optimal lobar treatment with $>20 \%$ low attenuation area and a visual CT assessment to verify the absence of airway disease were predictors of response to endobronchial coil [31].

Regarding safety, the two main serious adverse events reported were pneumonia and pneumothorax. In the RESET study, two pneumothoraces (5\%) occurred in the coil group, and none in the usual care group. No between-group difference was detected regarding serious adverse events. In the REVOLENS study, pneumonia was more frequent in the coil group ( $18 \%$ versus $4 \%$ ), whereas pneumothorax was not statistically more frequent (6\% versus $2 \%$ ) within 12 months. In the RENEW study, major complications were also more frequent in the coil group including pneumonia (20\% versus $4.5 \%)$ and pneumothorax (9.7\% versus $0.6 \%)$. The majority of pneumothoraces occurred during the first day post-coil treatment. Pneumonia occurred more frequently during the first month post-procedure. 
Overall, the three RCTs assessing coils showed similar results with significant improvement in hyperinflation, exercise tolerance and quality of life, with an acceptable safety profile. The risks of pneumonia and pneumothorax require pre-specified therapeutic management plans.

One limitation of the three RCTs is that no RCT included a sham group for control. The inclusion criteria in the three RCTs were relatively similar, selecting patients with severe airflow obstruction, homogeneous and heterogeneous emphysema, and severe hyperinflation. The primary end-points were somewhat heterogeneous regarding the time points with assessments at 3, 6 and 12 months. Results from short-time end-points have to be considered cautiously in emphysema. Primary end-points included quality of life in one study, and 6MWT results in two RCTs.

The GRADE level of evidence from the three RCTs is moderate with a low risk of bias, a low risk of imprecision, consistency in the efficacy results but limited to three RCTs, no evident indirectness, and no known publication bias.

\section{Long-term follow-up results}

Results beyond 1 year are available from two RCTs [32, 33] and one nonrandomised study [27].

A retrospective secondary analysis of the RESET study analysed 5-year survival after coil treatment [33]. The aim of this study was to assess 5-year overall and transplant-free survivals of patients treated by coils in the RESET study. The 5-year overall survival was 50.6\%, and the 5-year transplant-free survival was $46.7 \%$. Volume reduction responders at 3 months defined by a RV reduction of at least $10 \%$ had a higher 5 -year transplant-free survival than nonresponders.

2-year prospective follow-up data from 50 patients treated in the coil group in the REVOLENS showed sustained improvement from baseline in RV and SGRQ score, whereas changes in $\mathrm{FEV}_{1}$ and $6 \mathrm{MWT}$ were not significant [32]. Serious adverse events decreased after 1 year, with no unanticipated events occurring. No late pneumothorax or haemoptysis events were reported.

Data from 35 patients treated by coils in nonrandomised studies and who were invited for a voluntary annual visit at $1(n=35), 2(n=27)$ and 3 years $(n=22)$ have been reported [27]. A significant improvement from baseline was found for RV, mMRC scale and SGRQ score at 2 years, whereas the mMRC scale only was significantly improved at 3 years. The rate of responders at 3 years was $59 \%$ for the SGRQ score $(\leqslant 4$ points), $38 \%$ for $\mathrm{FEV}_{1}(\geqslant 10 \%), 19 \%$ for $\mathrm{RV}(\leqslant 400 \mathrm{~m})$, and $40 \%$ for $6 \mathrm{MWT}(\geqslant 26 \mathrm{~m})$. No late pneumothorax, coil migration or unexpected adverse event was reported beyond 1 year.

\section{Current recommendations}

The 2020 GOLD report indicates that coil treatment can be considered when available in both heterogeneous and homogeneous emphysema, with or without collateral ventilation (Evidence B) [29]. Recommendations from an international expert panel updated in 2019 recommended to consider coils in patients with heterogeneous or homogeneous emphysema with or without collateral ventilation, and with significant emphysema (low attenuation area $>20 \%$ at $950 \mathrm{HU}$ ), hyperinflation (RV $>225 \%$ and $\mathrm{RV} /$ total lung capacity $>0.58$ ), and no signs of significant airway wall thickening, bronchiectasis, or clinically significant chronic bronchitis [30].

\section{Thermal vapor ablation}

Thermal vapor ablation (TVA) (Uptake Medical Corporation, Seattle, WA, USA) is a bronchoscopic technique using instillation of heated water in the most destroyed part of upper lobes in a procedure usually performed under general anaesthesia or deep sedation [34]. Heated water locally induces an inflammatory response leading to parenchymal fibrosis and subsequent lung volume reduction. TVA has been assessed in one RCT.

\section{Levels of evidence}

The STEP-UP study is a multicentre study conducted in Germany, Austria, UK, Ireland and Australia, including 70 patients (TVA: $n=46$; usual care: $n=24$ ) [19]. The coprimary end-points were significantly improved in the TVA group compared to the control group for $\mathrm{FEV}_{1}$ and SGRQ scores at 6 months. Both $\mathrm{FEV}_{1}$ and SGRQ score remained significantly improved at 12 months, whereas RV and the 6MWT were not significantly different between the two groups [35]. A post hoc analysis of the STEP-UP study analysed the results for patients with collateral ventilation ( $78 \%$ of the patients treated by TVA), demonstrating as expected a significant improvement for $\mathrm{FEV}_{1}$ and SGRQ in this population [36]. These results highlight that TVA effectiveness is independent from collateral ventilation.

Regarding safety, COPD exacerbation occurred in $24 \%$ of the patients in the TVA group compared to $4 \%$ in the usual care group at 6 months [19]. One death was related to a COPD exacerbation occurring at day 
84, and was adjudicated as possibly related to TVA. No pneumothorax occurred within 30 days of treatment. Interestingly, the rate of serious adverse events was similar between both groups for the period 90-360 days [35].

The level of evidence for TVA relies on one RCT only, justifying conducting additional RCT in larger population to confirm these results.

The GRADE level of evidence for TVA relying on one RCT is low with a moderate risk of bias, a moderate risk of imprecision, absence of possibility to assess consistency because of available data from one RCT only, no evident indirectness, and no known publication bias.

Long-term follow-up results

No data beyond 1 year are currently available for TVA.

\section{Current recommendations}

The 2020 GOLD report indicates that TVA can be considered when available in heterogeneous emphysema (upper predominant), with or without collateral ventilation (Evidence B) [29]. Recommendations from an international expert panel updated in 2019 recommended considering TVA in upper lobe predominant heterogeneous emphysema only in clinical trials [30].

\section{Sealant}

The emphysematous lung sealant system (ELS) (PulmonX) employs a synthetic polymer delivered by fibreoptic bronchoscopy in upper lobe subsegments to induce remodelling and scar formation, therefore promoting atelectasis and reducing lung hyperinflation. Early nonrandomised studies showed promising results [37, 38] leading to the ASPIRE RCT [39]. Unfortunately, this RCT was terminated early for business reasons with 95 patients randomised out of 300 patients initially planned, and the primary 12-month end-point was then not assessed. Results at 3 months (57 patients) and 6 months (34 patients) suggested improvements in the ELS group in lung function, dyspnoea and quality of life [39]. Regarding safety, adverse events requiring hospitalisation were 2.5 -fold more frequent in the ELS group compared to controls, including pulmonary acute infiltratory response, COPD exacerbation, pneumonia and pneumothorax. Two deaths occurred in the ELS group.

The GRADE level of evidence for ELS is very low with a high risk of bias, a high risk of imprecision, an absence of possibility to assess consistency because of available data from one incomplete RCT only, indirectness, and no known publication bias.

Because of early termination of the only RCT, no definite conclusion can be drawn regarding efficacy and safety of ELS, thus requiring additional well-conducted RCTs.

\section{COPD}

Targeted lung denervation (TLD) is a novel bronchoscopic treatment aimed at attenuating parasympathetic overactivity in COPD by disrupting the peribronchial vagal innervation of the lung. Radiofrequency energy is delivered through a catheter with activation of the electrode in up to four positions in both main bronchi. The procedure is performed under general anaesthesia with bronchoscopic and fluoroscopic visualisation.

\section{Levels of evidence}

The first studies of TLD conducted in COPD assessed its feasibility and safety, and allowed optimal dosing and prevention and management of adverse events to be defined [40-42].

The AIRFLOW-2 is a multicentre sham bronchoscopy RCT conducted in symptomatic COPD patients $\left(\mathrm{mMRC} \geqslant 2\right.$ or COPD assessment test $\geqslant 10$ ) with $\mathrm{FEV}_{1} 30-60 \%$ predicted [20]. 82 patients were randomised to the TLD group with both lungs treated in a single procedure $(n=41)$ or to the sham group $(\mathrm{n}=41)$. The primary end-point was safety. The TLD group experienced fewer respiratory adverse events between 3 and 6.5 months post-TLD than the sham group. Secondary analyses showed that the rate of respiratory adverse events between 0 and 12.5 months post-TLD were not different between both groups, whereas the rate of COPD exacerbation requiring hospitalisation between 0 and 12.5 months post-TLD was lower in the TLD group. The overall number of serious adverse events was similar between both groups; however, there was a trend for increased gastrointestinal effects in the TLD group. Of note, there was no statistically significant difference in symptoms and lung function tests between groups over the 12.5 months of follow-up.

So far, results from only one RCT evaluating TLD in COPD have been published. The design of this RCT including a sham group and the results suggesting a reduction of respiratory events including COPD 
exacerbation requiring hospitalisation are interesting, but require additional RCTs from a larger population to confirm these findings. The AIRFLOW-3 study is an ongoing multicentre sham bronchoscopy RCT which should allow the ability to determine the efficacy and safety of TLD in COPD [43].

Because of the design of the only one RCT with a primary end-point for safety, it is difficult to assess the GRADE for TLD, thus requiring additional studies with efficacy primary end-points.

\section{Long-term follow-up results}

Data beyond 1 year are available from one prospective study including 15 patients treated on both lungs by TLD in a single procedure $(n=13)$ or two procedures $(n=2)$ [41]. Follow-up data are available for 15 patients at 1 year, 10 patients at 2 years, and nine patients at 3 years. The primary safety end-point was freedom from sustained worsening of COPD attributable to TLD up to 1 year, which was found in all the patients. Follow-up until 3 years was conducted in patients who reconsented for longer follow-up. Lung function and exercise capacity assessments conducted at 2 and 3 years post-TLD demonstrated similar beneficial effects of TLD without bronchodilators when compared with long-acting anticholinergic therapy.

12 serious adverse events including five respiratory events were reported through 3 years of follow-up, with no events related to TLD.

\section{Current recommendations}

The level of evidence is currently too low with available data from only one RCT to include TLD in therapeutic guidelines for COPD management. Additional RCTs are needed to confirm promising results, especially regarding the impact on severe COPD exacerbation.

\section{Conclusion}

The levels of evidence for interventional bronchoscopic techniques in asthma, COPD and emphysema largely improved in the past 15 years with many available RCTs results. To translate RCT results into clinical practice, these bronchoscopic techniques have to be considered in a multidisciplinary approach including precise assessment of asthma, COPD or emphysema phenotypes, comorbidities, as well as optimisation of medical management following international guidelines. Rigorous selection of the patients, experience and training in bronchoscopic procedures and management of potential adverse events are very important points to consider [44]. The risk benefit ratio has to be clearly discussed with the patients including potential efficacy, safety issues, and patient's preference. Data regarding long-term follow-up results beyond 1 year are currently sparse, especially in emphysema and COPD. The place of these bronchoscopic techniques in the global management of asthma, COPD and emphysema remains to be evaluated in clinical practice in real-life studies. Inclusion of patients in clinical studies or registries should be encouraged in order to obtain a higher level of evidence and long-term efficacy and safety data for each bronchoscopic technique.

Conflict of interest: J.M. Perotin has nothing to disclose. M. Dewolf has nothing to disclose. C. Launois has nothing to disclose. V. Dormoy has nothing to disclose. G. Deslee reports personal fees from BTG/PneumRx and Nuvaira, during the conduct of the study; personal fees from Chiesi, Boehringer Ingelheim, AstraZeneca and Novartis, outside the submitted work.

\section{References}

1 Cox PG, Miller J, Mitzner W, et al. Radiofrequency ablation of airway smooth muscle for sustained treatment of asthma: preliminary investigations. Eur Respir J 2004; 24: 659-663.

2 Danek CJ, Lombard CM, Dungworth DL, et al. Reduction in airway hyperresponsiveness to methacholine by the application of RF energy in dogs. J Appl Physiol 2004; 97: 1946-1953.

3 Laxmanan B, Egressy K, Murgu SD, et al. Advances in bronchial thermoplasty. Chest 2016; 150: 694-704.

4 Cox G, Thomson NC, Rubin AS, et al. Asthma control during the year after bronchial thermoplasty. N Engl J Med 2007; 356: 1327-1337.

5 Pavord ID, Cox G, Thomson NC, et al. Safety and efficacy of bronchial thermoplasty in symptomatic, severe asthma. Am J Respir Crit Care Med 2007; 176: 1185-1191.

6 Castro M, Rubin AS, Laviolette M, et al. Effectiveness and safety of bronchial thermoplasty in the treatment of severe asthma: a multicenter, randomized, double-blind, sham-controlled clinical trial. Am J Respir Crit Care Med 2010; 181: 116-124.

7 Thomson NC, Rubin AS, Niven RM, et al. Long-term (5 year) safety of bronchial thermoplasty: Asthma Intervention Research (AIR) trial. BMC Pulm Med 2011; 11: 8.

8 Wechsler ME, Laviolette M, Rubin AS, et al. Bronchial thermoplasty: long-term safety and effectiveness in patients with severe persistent asthma. J Allergy Clin Immunol 2013; 132: 1295-1302.

9 Davey C, Zoumot Z, Jordan S, et al. Bronchoscopic lung volume reduction with endobronchial valves for patients with heterogeneous emphysema and intact interlobar fissures (the BeLieVeR-HIFi study): a randomised controlled trial. Lancet 2015; 386: 1066-1073.

10 Klooster K, ten Hacken NH, Hartman JE, et al. Endobronchial valves for emphysema without interlobar collateral ventilation. N Engl J Med 2015; 373: 2325-2335. 
11 Valipour A, Slebos DJ, Herth F, et al. Endobronchial valve therapy in patients with homogeneous emphysema. results from the IMPACT Study. Am J Respir Crit Care Med 2016; 194: 1073-1082.

12 Kemp SV, Slebos DJ, Kirk A, et al. A multicenter randomized controlled trial of Zephyr endobronchial valve treatment in heterogeneous emphysema (TRANSFORM). Am J Respir Crit Care Med 2017; 196: 1535-1543.

13 Criner GJ, Sue R, Wright S, et al. A multicenter randomized controlled trial of Zephyr endobronchial valve treatment in heterogeneous emphysema (LIBERATE). Am J Respir Crit Care Med 2018; 198: 1151-1164.

14 Li S, Wang G, Wang $\mathrm{C}$, et al. The REACH trial: a randomized controlled trial assessing the safety and effectiveness of the Spiration valve system in the treatment of severe emphysema. Respiration 2019; 97: 416-427.

15 Criner GJ, Delage A, Voelker K, et al. Improving lung function in severe heterogenous emphysema with the Spiration valve system (EMPROVE). A multicenter, open-label randomized controlled clinical trial. Am J Respir Crit Care Med 2019; 200: 1354-1362.

16 Shah PL, Zoumot Z, Singh S, et al. Endobronchial coils for the treatment of severe emphysema with hyperinflation (RESET): a randomised controlled trial. Lancet Respir Med 2013; 1: 233-240.

17 Deslée G, Mal H, Dutau H, et al. Lung volume reduction coil treatment $v$ s usual care in patients with severe emphysema: the REVOLENS randomized clinical trial. JAMA 2016; 315: 175-184.

18 Sciurba FC, Criner GJ, Strange C, et al. Effect of endobronchial coils vs usual care on exercise tolerance in patients with severe emphysema: the RENEW randomized clinical trial. JAMA 2016; 315: 2178-2189.

19 Herth FJ, Valipour A, Shah PL, et al. Segmental volume reduction using thermal vapour ablation in patients with severe emphysema: 6-month results of the multicentre, parallel-group, open-label, randomised controlled STEP-UP trial. Lancet Respir Med 2016; 4: 185-193.

20 Slebos DJ, Shah PL, Herth FJF, et al. Safety and adverse events after targeted lung denervation for symptomatic moderate to severe chronic obstructive pulmonary disease (AIRFLOW). A multicenter randomized controlled clinical trial. Am J Respir Crit Care Med 2019; 200: 1477-1486.

21 Chupp G, Laviolette M, Cohn L, et al. Long-term outcomes of bronchial thermoplasty in subjects with severe asthma: a comparison of 3-year follow-up results from two prospective multicentre studies. Eur Respir J 2017; 50: 1700017 .

22 Chung KF, Wenzel SE, Brozek JL, et al. International ERS/ATS guidelines on definition, evaluation and treatment of severe asthma. Eur Respir J 2014; 43: 343-373.

23 Global Initiative for Asthma. Global Strategy for Asthma Management and Prevention, 2020. www.ginasthma.org

24 Zoumot Z, Davey C, Jordan S, et al. Endobronchial valves for patients with heterogeneous emphysema and without interlobar collateral ventilation: open label treatment following the BeLieVeR-HIFi study. Thorax 2017; 72: 277-279.

25 Klooster K, Hartman JE, Ten Hacken NH, et al. One-year follow-up after endobronchial valve treatment in patients with emphysema without collateral ventilation treated in the STELVIO Trial. Respiration 2017; 93: $112-121$

26 Garner J, Kemp SV, Toma TP, et al. Survival after endobronchial valve placement for emphysema: a 10-year follow-up study. Am J Respir Crit Care Med 2016; 194: 519-521.

27 Hartman JE, Klooster K, Gortzak K, et al. Long-term follow-up after bronchoscopic lung volume reduction treatment with coils in patients with severe emphysema. Respirology 2015; 20: 319-326.

28 Gompelmann D, Heinhold T, Rötting M, et al. Long-term follow up after endoscopic valve therapy in patients with severe emphysema. Ther Adv Respir Dis 2019; 13: 1753466619866101.

29 Global Initiative for Chronic Obstructive Lung Disease. Global strategy for the diagnosis, management and prevention of chronic obstructive pulmonary disease. 2020. https://goldcopd.org/gold-reports

30 Herth FJF, Slebos DJ, Criner GJ, et al. Endoscopic lung volume reduction: an expert panel recommendation update 2019. Respiration 2019; 97: 548-557.

31 Slebos DJ, Cicenia J, Sciurba FC, et al. Predictors of response to endobronchial coil therapy in patients with advanced emphysema. Chest 2019; 155: 928-937.

32 Deslée G, Leroy S, Perotin JM, et al. Two-year follow-up after endobronchial coil treatment in emphysema: results from the REVOLENS study. Eur Respir J 2017; 50: 1701740.

33 Garner JL, Kemp SV, Srikanthan K, et al. 5-year survival after endobronchial coil implantation: secondary analysis of the first randomised controlled trial, RESET. Respiration 2020: 99; 154-162.

34 Gompelmann D, Shah PL, Valipour A, et al. Bronchoscopic thermal vapor ablation: best practice recommendations from an expert panel on endoscopic lung volume reduction. Respiration 2018; 95: 392-400.

35 Shah PL, Gompelmann D, Valipour A, et al. Thermal vapour ablation to reduce segmental volume in patients with severe emphysema: STEP-UP 12-month results. Lancet Respir Med 2016; 4: e44-e45.

36 Gompelmann D, Eberhardt R, Schuhmann M, et al. Lung volume reduction with vapor ablation in the presence of incomplete fissures: 12-month results from the STEP-UP randomized controlled study. Respiration 2016; 92: $397-403$.

37 Kramer MR, Refaely Y, Maimon N, et al. Bilateral endoscopic sealant lung volume reduction therapy for advanced emphysema. Chest 2012; 142: 1111-1117.

38 Herth FJ, Gompelmann D, Stanzel F, et al. Treatment of advanced emphysema with emphysematous lung sealant $\left(\right.$ AeriSeal $\left.^{\circ}\right)$. Respiration 2011; 82: 36-45

39 Come CE, Kramer MR, Dransfield MT, et al. A randomised trial of lung sealant versus medical therapy for advanced emphysema. Eur Respir J 2015; 46: 651-662.

40 Slebos DJ, Klooster K, Koegelenberg CF, et al. Targeted lung denervation for moderate to severe COPD: a pilot study. Thorax 2015; 70: 411-419.

41 Valipour A, Asadi S, Pison C, et al. Long-term safety of bilateral targeted lung denervation in patients with COPD. Int J Chron Obstruct Pulmon Dis 2018; 13: 2163-2172.

42 Valipour A, Shah PL, Pison C, et al. On behalf of the AIRFLOW-1 study group. Safety and dose study of targeted lung denervation in moderate/severe COPD patients. Respiration 2019; 98: 329-339.

43 Slebos DJ, Degano B, Valipour A, et al. Design for a multicenter, randomized, sham-controlled study to evaluate safety and efficacy after treatment with the Nuvaira lung denervation system in subjects with chronic obstructive pulmonary disease (AIRFLOW-3). BMC Pulm Med 2020; 20: 41.

44 Deslee G. Endobronchial lung volume reduction in severe emphysema. time to translate randomized controlled trial results into routine clinical practice? Am J Respir Crit Care Med 2018; 198: 1110-1112. 